\title{
Corrigendum: Small cell carcinoma of the ovary, hypercalcemic type, displays frequent inactivating germline and somatic mutations in SMARCA4
}

Pilar Ramos, Anthony N Karnezis, David W Craig, Aleksander Sekulic, Megan L Russel, William P D Hendricks, Jason J Corneveaux, Michael T Barrett, Karey Shumansky, Yidong Yang, Sohrab P Shah, Leah M Prentice, Marco A Marra, Jeffrey Kiefer, Victoria L Zismann, Troy A McEachron, Bodour Salhia, Jaime Prat, Emanuela D’Angelo, Blaise A Clarke, Joseph G Pressey, John H Farley, Stephen P Anthony, Richard B S Roden, Heather E Cunliffe, David G Huntsman \& Jeffrey M Trent

Nat. Genet. 46, 427-429 (2014); published online 23 March 2014; corrected after print 13 June 2014

In the version of this article initially published, there were errors in the numbers of tumors reported in the text. The abstract reports SMARCA4 mutations in $75 \%$ (9/12) of tumors. The correct numbers are $69 \%(9 / 13)$ of tumors. The main text reports immunohistochemical analysis of 15 tumors, with 6 tumors overlapping with the 12 cases sequenced. These should have been reported as 17 tumors, 8 of which were overlapping. The errors do not affect any of the analyses or conclusions in the paper. In addition, a secondary affiliation for author Pilar Ramos was erroneously omitted and should read "School of Life Sciences, Arizona State University, Tempe, Arizona, USA." The errors have been corrected in the HTML and PDF versions of the article. 